\title{
Eccentric Cycling Training Improves Health-Related Quality of Life in Adolescents with Obesity
}

\author{
Valérie Julian $^{a}$ David Thivel $^{\mathrm{b}}$ Maud Miguet $^{\mathrm{b}}$ Bruno Pereira $^{\mathrm{c}}$ \\ Céline Lambert $^{c}$ Frédéric Costes ${ }^{a}$ Ruddy Richard ${ }^{a}$ Martine Duclos ${ }^{a}$ \\ ${ }^{a}$ Department of Sport Medicine and Functional Explorations, University Teaching \\ Hospital of Clermont-Ferrand, CRNH, INRA, University of Clermont Auvergne, \\ Clermont-Ferrand, France; 'baboratory AME2P, University of Clermont Auvergne, \\ Aubière, France; ' Department of Biostatistics, University Teaching Hospital of \\ Clermont-Ferrand, Clermont-Ferrand, France
}

\section{Keywords}

Childhood obesity · Quality of life · Physical activity · Eccentric training · Concentric training

\begin{abstract}
Introduction: While eccentric (ECC) training appears to be more efficient than concentric (CON) training at improving body composition in adolescent with obesity, its impact on health-related quality of life (HRQOL) has never been studied. Objective: The aim of this study is to compare the effects of 2 cycling training modalities, i.e., ECC vs. CON, in adolescents with obesity on HRQOL and health perception (HP). Methods: A total of 24 adolescents with obesity, aged 12-16 years, were randomized to either a 12-week ECC or a CON cycling training program performed at the same oxygen consumption $\left(\mathrm{VO}_{2}\right)$. Anthropometric measurements, body composition, maximal incremental tests, HRQOL (Vécu et Santé Percue de l'Adolescent [VSP-A], Medical Outcome Study Short Form [SF-36]), and HP were assessed at before and after training. Results and Conclusion: Both CON and ECC cycling trainings promoted significant improvements in $\mathrm{BMI}, \mathrm{VO}_{2 \text { peak, }}$, total fat mass, and fat-free mass, with better improvements in body composition parameters in the ECC group $(p<0.05)$. The VSP-A total score increased after CON $(p<0.01)$ and ECC $(p<0.001)$ training, with better enhancement for the ECC group $(p<0.05)$. The SF-36 physical score increased after both CON $(p<0.01)$ and ECC $(p<0.001)$ trainings. The global HP score increased only after ECC training $(p<0.001)$. Except for the energy-vitality item, no significant correlation was found between changes in HRQOL and its subdomains and anthropometric, body composition, and functional parameters. Both ECC and CON cycling trainings are associated with positive changes in HRQOL and HP. However, ECC seems to induce greater improvements in HRQL and HP than CON cycling training, which is probably not due to the anthropometric, body composition, and functional changes.




\section{Introduction}

The worldwide prevalence of obesity is alarming, with approximately $80 \%$ of adolescents with obesity likely to remain obese during adulthood [1]. Pediatric obesity results in metabolic complications and cardiac, respiratory, or muscular limitations to exercise capacities [1]. Cognitive and psychosocial impairments, such as increased anxiety, and reduced self-esteem, social integration, and academic performance, have also been observed in children with obesity [2]. Pediatric obesity is associated with a reduced health-related quality of life (HRQOL) [2], since youth with obesity are approximately 5 times more likely to show a reduced HRQOL compared to lean individuals [3].

Lifestyle interventions and physical training are now considered effective strategies to improve obesity indicators, metabolic complications, functional impairments, psychological morbidities, and HRQOL in children with obesity [4-7]. Negative associations between HRQOL and obesity indicators, such as body weight, BMI, and fat mass percentage, have been reported $[2,5,8]$. Multidisciplinary interventions have been mainly used to induce weight loss and they have been shown to improve HRQOL in patients with obesity [2, 5-7]. For the last 10-15 years, much research has been conducted to identify the best exercise modality to improve weight loss programs, such as high-intensity interval training, concurrent training, moderate-intensity continuous training, and others. While the body of evidence is now consistent regarding the anthropometric, physiological, and metabolic effects of such modalities, their role in HRQOL has not been properly assessed yet in adolescents with obesity. Recently, studies have focused on the use of eccentric (ECC) exercises, over the common concentric (CON) modality, as an efficient antiobesity strategy in both adults and youth with obesity [9-11]. ECC exercise, characterized by a lower metabolic demand (i.e., low oxygen consumption) and muscle activity compared to CON exercise for a similar level of exerted force, represents an optimal modality for patients with chronic pathologies [12,13], particularly for patients with overweight or obesity. Our team recently showed that ECC cycling training is twice more efficient than CON training for decreasing whole-body fat mass and increasing the lean mass in adolescents with obesity, while inducing greater strength gains and insulin-resistance improvements than CON training when performed at the same metabolic rate [10]. Although ECC is an efficient strategy to induce weight loss and improve the metabolic profile in adolescents with obesity, its effect on HRQOL remains unknown.

The main aim of the present work was to compare the effects of 12-week ECC versus CON cycling performed at the same metabolic rate on HRQOL in adolescents with obesity. The secondary aim was to compare the impact of these 2 modalities of exercise training on health perception (HP) and to investigate the correlations between HRQOL and anthropometric, metabolic, and functional parameters.

\section{Materials and Methods}

\section{Study Participants}

Twenty-four adolescents (Tanner stages 3-4) of both genders were included in this study (Children Medical Centre for Obese Adolescents of La Bourboule, France). The patients met the following criteria: (1) age 12-16 years; (2) BMI >90th percentile, according to the international cut-off points [14]; (3) no medication affecting energy metabolism and no regular tobacco or alcohol use; and (4) no contraindication for exercise. 


\section{Study Design and Training Program}

After undergoing a medical examination to ensure their ability to complete this study, adolescents were randomized and assigned to either a CON or an ECC group. Anthropometric, body composition, and functional parameters, as well as HRQOL and HP, were assessed before and after the 12-week training intervention period.

The intervention has been described in detail previously [10]. In brief, for the 2 groups, the training program consisted of a 12-week physical activity program with 36 sessions ( 3 sessions per week). The training contained the following 3 phases: (1) phase 1, a 2-week habituation period (i.e., a progressive increase in exercise intensity and the duration of the sessions) to protect individuals from delayed-onset muscle soreness; (2) phase 2, fortyminute sessions with ECC or CON cycling at $50 \% \mathrm{VO}_{2 \text { peak }}$ during 5 weeks, and (3) phase 3 , forty-minute sessions with ECC or CON cycling at $70 \% \mathrm{VO}_{2 \text { peak }}$ during 5 weeks. ECC training was performed on ECC motor-driven ergometers (Cyclus2 Eccentric Recumbent; MSE Medical, France) and CON training on classical CON ergometers (Optibike Med 600; MSE Medical, France). A physical therapist and an exercise physiologist supervised all of the training sessions. All of the participants received the same nutritional education. The diet was controlled, with no dietary restrictions per se. The adolescents followed a balanced normocaloric diet provided and supervised by the Pediatric Obesity Center, according to the national recommendations for adolescents (Plan National Nutrition Santé) depending on their age range and gender (i.e., 12-15 years, from 40 to $50 \mathrm{kcal} / \mathrm{kg} /$ day). The mean daily composition of macronutrients was 35\% lipids, 55\% carbohydrates, and 15\% proteins (not exceeding 0.9 $\mathrm{g} / \mathrm{kg} /$ day) [10].

\section{Anthropometric, Body Composition, and Functional Evaluations}

Weight and height were measured to an accuracy of $0.1 \mathrm{~kg}$ and $0.5 \mathrm{~cm}$. Body composition was evaluated using dual-energy X-ray absorptiometry (DXA, Hologic QDR-4500A, Bedford, MA, USA) by a trained blinded technician. Data were analyzed using Hologic QDR software for Windows (version 12.6) [10].

$\mathrm{VO}_{2 \text { peak }}$ was evaluated for each individual during a maximal incremental CON exercise test. The initial power was set at $30 \mathrm{~W}$ for $3 \mathrm{~min}$, followed by a 15-W increment every minute until exhaustion. Adolescents were strongly encouraged by the supervisors throughout the test to perform a maximal effort. The maximal exercise test was defined by at least 2 of the following criteria: heart rate $>90 \%$ of the theoretical maximum heart rate $(210-0.65 \times$ age $)$, respiratory exchange ratio $\left(\mathrm{CVO}_{2} / \mathrm{VO}_{2}\right)>1.1$, and $\mathrm{VO}_{2}$ plateau [10]. Cardiac electrical activity was monitored (Ultima Series ${ }^{\mathrm{TM}}$; Saint Paul, MN, USA) and the test was coupled with measurement of gas exchanges breath by breath (BreezeSuite Software; Saint Paul, MN, USA) to determine the $\mathrm{VO}_{2}$ production. $\mathrm{VO}_{2 \text { peak }}$ was defined as the average $\mathrm{VO}_{2}$ of the last $30 \mathrm{~s}$ of exercise before exhaustion [10].

\section{Health-Related Quality of Life}

Vécu et Santé Percue de l'Adolescent Questionnaire

The Vécu et Santé Percue de l'Adolescent (VSP-A) questionnaire is a French generic selfadministered HRQOL questionnaire for adolescents aged 11-17 years, which is capable of discriminating between ill and healthy adolescents $[15,16]$. It is compiled of 39 separate questions, which should be completed in about $15 \mathrm{~min}$. Each question is coupled with 5 types of response, i.e., never-not at all, rarely-a bit, sometimes-moderately, often-a lot, and always, numbered as $0,25,50,75$, or 100 in ascending order of quality of life. The following 10 dimensions are tested: psychological well-being (5 questions), body image (2 questions), relationship with friends ( 5 questions), leisure (4 questions), relationship with parents (4 questions), relationship with teachers (3 questions), physical well-being (4 questions),

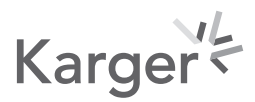


vitality ( 5 questions), school performance ( 2 questions), and relationship with medical staff (3 questions) [15]. Our interpretation considered the overall score and the score for each dimension.

Medical Outcome Study Short Form Questionnaire

The Medical Outcome Study Short Form (SF-36) is one of the most widely used selfadministered QoL questionnaires; it is often referred to as the golden standard [17] and it is completed in approximately $15 \mathrm{~min}$. It is a 36-item multipurpose, short-form health survey. People are invited to recall the past 4 weeks. The response categories vary depending on the question. These range from 2 (yes or no) to 6 (all of the time, most of the time, a good bit of the time, some of the time, a little of the time, or none of the time). Subscale scores range from 0 to 100 in ascending order of quality of life. Questions are grouped into 8 dimensions, i.e., physical functioning (PF; 10 questions), limitations in usual role activities because of physical aspects (role physical [RF], 4 questions), bodily pain (BP; 2 questions), general HP (GH; 6 questions), vitality (VT; energy and fatigue, 4 questions), social functioning (SF; 2 questions), limitation in usual role activities because of emotional problems (role emotional [RE], 3 questions) and mental health (MH; 5 questions). From these 8 dimensions, it is possible to calculate 2 global scores, i.e., a physical score (PS; which gathers PF, RB, BP, and GH dimensions), and a mental score (MS; which gathers VT, SF, RE, and MH dimensions).

\section{HP Questionnaire}

The HP questionnaire is a short self-administered questionnaire specifically designed to explore the perception of health, which refers to "how people consider their own health." It has been previously validated in adults $[18,19]$ and used in children with obesity [20]. The HP scale investigates the following 6 criteria: perceived physical fitness, perceived ideal weight, perceived healthy balanced diet, perceived sleep quality, perceived stress level, and perceived general health. Each item is evaluated using a 10-point scale from 1 (not at all) to 10 (very much). These 6 individual scores were considered for the global HP score. Adolescents were asked to respond alone to all questionnaires; however, a medical doctor was present to exclude misunderstandings.

\section{Statistical Analysis}

Because this work is an ancillary study of a broader project [9], the sample size was not initially calculated based on HRQOL, which was a secondary outcome. However, with 11 individuals for each randomized group, a minimal effect size equal to 1.2 can be calculated for a 2 -sided type 1 error at $5 \%$ and a statistical power $>80 \%$.

Statistical analyses were performed using Stata software, version 13 (StataCorp, College Station, TX, USA). Tests were 2 -sided, with a type 1 error set at $5 \%$. Continuous data were expressed as means \pm SD or medians (IQR) depending on the statistical distribution. The assumption of normality was assessed using the Shapiro-Wilk test.

Participants' age was compared between groups (CON vs. ECC) using a Student $t$ test. Homoscedasticity was analyzed using the Fisher-Snedecor test. Categorical parameters were compared between groups using a $\chi^{2}$ test or a Fisher exact test. Relationships between continuous variables were studied by estimating Pearson or Spearman correlation coefficients, depending on the statistical distribution. They were interpreted according to the following rules of thumb: $0.00-0.10$, negligible correlation; $0.10-0.39$, weak correlation; $0.40-0.69$, moderate correlation; $0.70-0.89$, strong correlation; and $0.90-1.00$, very strong correlation. A Sidak type 1 error correction was applied for multiple comparisons.

For correlated repeated measures, random-effects models were performed to measure time and group (CON vs. ECC) effects and their interaction (time $\times$ group), taking into account

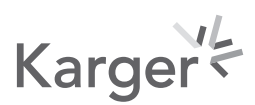


Julian et al.: ECC Training and Quality of Life

Table 1. Anthropometric measurements, body composition, and functional parameters before and after the 12-week cycling program for the CON and ECC groups in adolescents with obesity

\begin{tabular}{|c|c|c|c|c|c|}
\hline & \multicolumn{2}{|c|}{$\operatorname{CON}(n=12)$} & \multicolumn{2}{|l|}{$\operatorname{ECC}(n=11)$} & \multirow{2}{*}{$\begin{array}{l}p \text { value } \\
\text { time } \times \text { group } \\
\text { interaction }\end{array}$} \\
\hline & before & after & before & after & \\
\hline Weight, kg & $76.6 \pm 12.1$ & $74.1 \pm 11.5^{* * *}$ & $82.8 \pm 17.4$ & $78.8 \pm 16.0^{* * *}$ & 0.739 \\
\hline Height, cm & $159.6 \pm 7.7$ & $161.5 \pm 7.5^{* *}$ & $163.4 \pm 7.2$ & $164.5 \pm 7.6^{* *}$ & 0.849 \\
\hline BMI & $30.0 \pm 3.5$ & $28.4 \pm 3.7^{* * *}$ & $30.8 \pm 4.9$ & $29.0 \pm 4.5^{* * *}$ & 0.859 \\
\hline Whole-body lean mass, $\%$ & $65.6 \pm 4.8$ & $66.6 \pm 6.0^{*}$ & $67.2 \pm 4.8$ & $69.7 \pm 6.3^{* *}$ & $0.041^{\dagger}$ \\
\hline Whole-body fat, $\%$ & $31.8 \pm 5.1$ & $30.7 \pm 6.3^{*}$ & $30.3 \pm 5.1$ & $27.5 \pm 6.6^{* * *}$ & $0.038^{\dagger}$ \\
\hline $\mathrm{VO}_{2 \text { peak }}, \mathrm{L} \times \min ^{-1}$ & $2,133 \pm 472$ & $2,277 \pm 473^{* *}$ & $2,297 \pm 590$ & $2,496 \pm 499 * *$ & 0.387 \\
\hline Isometric peak torque & $150.3 \pm 29.7$ & $156.3 \pm 31.1$ & $147.1 \pm 33.9$ & $181.5 \pm 17.8^{* * *}$ & $0.036^{\dagger}$ \\
\hline
\end{tabular}

Values are presented as means $\pm \mathrm{SD}$. The total number of subjects is $23 .{ }^{*} p<0.05 .{ }^{* *} p<0.01 .{ }^{* *} p<0.001$. Time $\times$ group interaction ${ }^{\dagger} p<0.05$.

Table 2. VSP-A scores before and after the 12-week cycling program for the CON and ECC groups in adolescents with obesity $(n=23$; mean $\pm \mathrm{SD})$

\begin{tabular}{|c|c|c|c|c|c|}
\hline & \multicolumn{2}{|c|}{$\operatorname{CON}(n=12)$} & \multicolumn{2}{|c|}{$\operatorname{ECC}(n=11)$} & \multirow{2}{*}{$\begin{array}{l}p \text { value } \\
\text { time } \times \text { group } \\
\text { interaction }\end{array}$} \\
\hline & before & after & before & after & \\
\hline Total VSP-A & $54.7 \pm 8.46$ & $61.5 \pm 10.2^{* *}$ & $57.2 \pm 13.3$ & $73.9 \pm 17.3^{* * *}$ & $0.027^{\dagger}$ \\
\hline Psychological well-being & $61.3 \pm 23.3$ & $79.2 \pm 21.9 *$ & $62.7 \pm 28.4$ & $75.0 \pm 23.2^{*}$ & 0.592 \\
\hline Body image & $37.5 \pm 25.6$ & $67.7 \pm 32.6^{* *}$ & $44.3 \pm 16.2$ & $78.4 \pm 23.1^{* * *}$ & 0.720 \\
\hline Friends relationships & $64.6 \pm 25.5$ & $68.3 \pm 16.0$ & $71.8 \pm 22.6$ & $80.0 \pm 18.6$ & 0.606 \\
\hline Leisure & $57.8 \pm 24.2$ & $67.2 \pm 23.1$ & $67.6 \pm 22.8$ & $65.3 \pm 30.9$ & 0.234 \\
\hline Parents relationships & $60.4 \pm 24.9$ & $65.1 \pm 18.7$ & $60.2 \pm 25.2$ & $72.2 \pm 22.8^{*}$ & 0.312 \\
\hline Teachers relationships & $33.3 \pm 23.0$ & $47.2 \pm 32.8$ & $41.5 \pm 16.4$ & $73.5 \pm 23.8^{* * *}$ & 0.091 \\
\hline Physical well-being & $49.5 \pm 16.3$ & $59.9 \pm 17.5$ & $45.6 \pm 14.7$ & $74.4 \pm 13.8^{* * *}$ & $0.035^{\dagger}$ \\
\hline Energy-vitality & $66.3 \pm 18.5$ & $58.3 \pm 22.0$ & $64.5 \pm 17.8$ & $78.2 \pm 24.5^{* *}$ & $0.011^{\dagger}$ \\
\hline School performance & $47.9 \pm 25.5$ & $45.8 \pm 20.9$ & $46.6 \pm 25.1$ & $61.4 \pm 26.5$ & 0.192 \\
\hline Medical staff relationship & $68.8 \pm 28.9$ & $56.3 \pm 30.8$ & $79.5 \pm 18.4$ & $81.1 \pm 21.8$ & 0.297 \\
\hline
\end{tabular}

Values are presented as means \pm SD. The total number of patients is 23 . $^{*} p<0.05$. $^{* *} p<0.01$. $^{* * *} p<0.001$. Time $\times$ group interaction ${ }^{\dagger} p<0.05$.

between- and within-participant variability (subject as a random effect). The normality of residuals from these models was studied using the Shapiro-Wilk test. When appropriate, a logarithmic transformation was proposed to achieve normality of dependent outcomes. As mentioned before, Sidak type 1 error correction was applied for 2-by-2 comparisons.

\section{Results}

Twenty-three adolescents completed this study. One adolescent in the ECC group dropped out due to family reasons, resulting in 11 subjects ( 6 girls and 5 boys) in the ECC group and 12 in the CON group ( 6 boys and 6 girls). The mean age of the adolescents was $13.3 \pm 1.2$ years in the CON group and $13.6 \pm 1.3$ years in the ECC group $(p=0.49)$. The results of anthropometric, body composition, and functional parameters $\left(\mathrm{VO}_{2 \text { peak }}\right.$ and quadriceps strength) 


\begin{tabular}{|c|c|c|c|c|c|c|c|c|}
\hline \multirow{12}{*}{$\begin{array}{l}\frac{\pi}{1} \\
\text { क }\end{array}$} & \multicolumn{2}{|l|}{-1} & -0.5 & \multirow{2}{*}{$\begin{array}{c}\mathbf{0} \\
-0.09 \\
\end{array}$} & \multicolumn{2}{|c|}{0.5} & 1 & \multirow{12}{*}{$\begin{array}{l}\text { Total VSP-A } \\
\text { Psychological well being } \\
\text { Body image } \\
\text { Friends relationships } \\
\text { Leisure } \\
\text { Parents relationships } \\
\text { Teachers relationships } \\
\text { Physical well-being } \\
\text { Energy-vitality } \\
\text { School performance } \\
\text { Medical staff relationship }\end{array}$} \\
\hline & -0.20 & -0.29 & -0.23 & & -0.09 & 0.02 & 0.10 & \\
\hline & 0.11 & 0.12 & 0.25 & 0.41 & -0.18 & 0.11 & 0.23 & \\
\hline & -0.31 & -0.21 & -0.02 & 0.13 & -0.48 & 0.21 & 0.14 & \\
\hline & 0.33 & 0.31 & 0.22 & 0.01 & 0.37 & -0.27 & -0.21 & \\
\hline & 0.07 & -0.12 & 0.15 & 0.20 & -0.07 & 0.01 & -0.54 & \\
\hline & 0.35 & 0.46 & 0.31 & 0.04 & 0.15 & -0.13 & 0.19 & \\
\hline & -0.27 & -0.29 & -0.30 & -0.11 & 0.09 & -0.12 & -0.04 & \\
\hline & -0.05 & 0.05 & -0.11 & -0.32 & -0.07 & 0.22 & 0.33 & \\
\hline & -0.64 & -0.63 & -0.53 & -0.26 & -0.35 & -0.04 & 0.12 & \\
\hline & -0.19 & -0.32 & -0.13 & -0.09 & -0.14 & 0.20 & 0.22 & \\
\hline & -0.03 & -0.24 & -0.25 & -0.15 & 0.34 & 0.20 & 0.07 & \\
\hline \multirow{10}{*}{$\begin{array}{l}\stackrel{0}{\tilde{L}} \\
\stackrel{\tilde{\omega}}{ }\end{array}$} & -0.11 & -0.08 & -0.27 & -0.25 & 0.02 & -0.02 & 0.29 & \multirow{10}{*}{$\begin{array}{l}\text { Physical functioning } \\
\text { Role physical } \\
\text { Bodily Pain } \\
\text { General Health } \\
\text { Physical score } \\
\text { Vitality } \\
\text { Social Functioning } \\
\text { Role emotional } \\
\text { Mental Health } \\
\text { Social and mental score }\end{array}$} \\
\hline & 0.09 & 0.10 & 0.42 & 0.55 & -0.22 & 0.08 & -0.14 & \\
\hline & 0.20 & 0.22 & 0.37 & 0.28 & 0.07 & -0.02 & 0.15 & \\
\hline & 0.00 & 0.13 & 0.43 & 0.43 & -0.43 & -0.19 & 0.26 & \\
\hline & 0.30 & 0.34 & 0.62 & 0.53 & -0.13 & $\begin{array}{l}-0.07 \\
\end{array}$ & -0.04 & \\
\hline & -0.07 & 0.00 & 0.30 & 0.32 & -0.32 & $\begin{array}{l}-0.04 \\
\end{array}$ & 0.01 & \\
\hline & 0.09 & 0.00 & 0.13 & 0.11 & 0.12 & -0.21 & -0.25 & \\
\hline & 0.12 & 0.10 & 0.04 & -0.05 & 0.23 & 0.04 & -0.07 & \\
\hline & -0.28 & -0.20 & 0.05 & 0.24 & -0.30 & -0.14 & 0.05 & \\
\hline & -0.07 & -0.10 & 0.11 & 0.23 & -0.04 & 0.02 & -0.14 & \\
\hline \multirow{8}{*}{ 呈 } & 0.06 & -0.16 & -0.11 & 0.01 & 0.19 & 0.21 & 0.06 & \multirow{8}{*}{$\begin{array}{l}\text { Physical Condition } \\
\text { Adiposity } \\
\text { Alimentation } \\
\text { Sleep } \\
\text { Stress } \\
\text { General Health } \\
\text { Global score of perceived Health }\end{array}$} \\
\hline & 0.17 & 0.17 & 0.37 & 0.34 & -0.30 & 0.11 & -0.37 & \\
\hline & 0.40 & 0.21 & 0.40 & 0.19 & 0.23 & -0.21 & -0.40 & \\
\hline & 0.16 & 0.02 & 0.18 & 0.18 & 0.06 & 0.12 & -0.22 & \\
\hline & -0.06 & -0.09 & -0.11 & -0.17 & -0.03 & 0.08 & 0.39 & \\
\hline & 0.02 & -0.04 & 0.03 & 0.01 & 0.08 & -0.14 & -0.14 & \\
\hline & 0.23 & 0.03 & 0.20 & 0.11 & 0.16 & 0.10 & 0.23 & \\
\hline & $\begin{array}{l}\text { Body } \\
\text { weight }\end{array}$ & BMI & FM (kg) & FM (\%) & FFM & VO2peak & Torque & \\
\hline
\end{tabular}

Fig. 1. Heatmap representation of the correlations between each item of quality of life and HP variables and anthropometric, body composition, and functional variables between pre- and posttraining. The darker the box, the higher the correlation.

before and after the training program have been previously published [9] and are summarized in Table 1.

Total VSP-A ( $p<0.001$ in the ECC group and $p<0.01$ in the CON group), the psychological well-being score ( $p<0.05$ in both groups), and body image scores $(p<0.001$ in the ECC group and $p<0.01$ in the CON group) increased significantly in both groups after exercise training. Physical well-being $(p<0.001)$, energy-vitality $(p<0.01)$, and parent $(p<0.05)$ and teacher $(p<0.001)$ relationship scores increased significantly only in the ECC group. A better improvement of total VSP-A was observed in the ECC group compared to the CON group ( $p=$ 0.03). Data related to the changes in VSP-A scores are summarized in Table 2. Correlation tests revealed only moderate correlations between the increase in energy-vitality and the decrease in weight (coefficient correlation $=0.637 ; p=0.002$ ), BMI (coefficient correlation $=$ $-0.630 ; p=0.002$ ), and fat mass (coefficient correlation $=-0.523 ; p=0.013$ ). All correlations are presented in Figure 1. 
Table 3. SF-36 scores before and after the 12-week cycling program for the CON and ECC groups in adolescents with obesity

\begin{tabular}{|c|c|c|c|c|c|}
\hline & \multicolumn{2}{|c|}{$\operatorname{CON}(n=12)$} & \multicolumn{2}{|c|}{$\operatorname{ECC}(n=11)$} & \multirow{2}{*}{$\begin{array}{l}p \text { value } \\
\text { time } \times \text { group } \\
\text { interaction }\end{array}$} \\
\hline & before & after & before & after & \\
\hline $\mathrm{PF}$ & $85.0 \pm 25.6$ & $95.8 \pm 5.1$ & $85.9 \pm 20.1$ & $95.9 \pm 5.8^{*}$ & 0.920 \\
\hline $\mathrm{RP}$ & $70.8 \pm 29.8$ & $85.4 \pm 22.5$ & $70.4 \pm 24.5$ & $82.5 \pm 26.4^{* * *}$ & 0.897 \\
\hline $\mathrm{BP}$ & $84.8 \pm 21.6$ & $86.9 \pm 15.8$ & $89.8 \pm 13.5$ & $91.0 \pm 13.8$ & 0.515 \\
\hline GH & $56.5 \pm 13.8$ & $69.1 \pm 17.1^{* *}$ & $67.0 \pm 21.0$ & $74.2 \pm 16.4^{* *}$ & 0.511 \\
\hline PS & $73.3 \pm 13.5$ & $84.3 \pm 11.5^{* *}$ & $78.3 \pm 14.0$ & $85.9 \pm 10.2^{* * *}$ & 0.589 \\
\hline VT & $59.6 \pm 7.5$ & $54.6 \pm 9.4$ & $61.3 \pm 17.5$ & $58.0 \pm 13.8$ & 0.720 \\
\hline SF & $72.9 \pm 21.2$ & $80.2 \pm 18.0$ & $81.8 \pm 21.9$ & $80.0 \pm 21.4$ & 0.395 \\
\hline $\mathrm{RE}$ & $72.2 \pm 27.8$ & $83.3 \pm 25.6$ & $75.7 \pm 36.7$ & $93.3 \pm 14.1$ & 0.583 \\
\hline $\mathrm{MH}$ & $65.7 \pm 10.7$ & $62.3 \pm 16.6$ & $65.8 \pm 15.2$ & $68.8 \pm 20.1$ & 0.106 \\
\hline Social and mental score & $67.6 \pm 9.5$ & $70.1 \pm 13.1$ & $71.2 \pm 16.3$ & $75.0 \pm 9.7$ & 0.650 \\
\hline
\end{tabular}

Values are presented as means $\pm \mathrm{SD}$. The total number of subjects is $23 .{ }^{*} p<0.05 .{ }^{* *} p<0.01 .{ }^{* * *} p<0.001$.

Table 4. Health perception scores before and after the 12-week cycling program for the CON and ECC groups in adolescents with obesity

\begin{tabular}{|c|c|c|c|c|c|}
\hline & \multicolumn{2}{|c|}{$\operatorname{CON}(n=12)$} & \multicolumn{2}{|c|}{$\operatorname{ECC}(n=11)$} & \multirow{2}{*}{$\begin{array}{l}p \text { value } \\
\text { time } \times \text { group } \\
\text { interaction }\end{array}$} \\
\hline & before & after & before & after & \\
\hline Physical condition & $5.08 \pm 1.73$ & $6.42 \pm 1.73^{*}$ & $5.27 \pm 1.49$ & $7.45 \pm 1.37^{* * *}$ & 0.217 \\
\hline Adiposity & $7.50 \pm 1.98$ & $7.00 \pm 1.46$ & $6.73 \pm 1.10$ & $6.55 \pm 0.93$ & 0.208 \\
\hline Alimentation & $5.41 \pm 2.64$ & $6.92 \pm 2.35$ & $7.27 \pm 1.79$ & $7.18 \pm 1.83$ & 0.033 \\
\hline Sleep & $6.50 \pm 3.37$ & $5.92 \pm 3.23$ & $7.00 \pm 2.49$ & $7.45 \pm 2.34$ & 0.255 \\
\hline Stress & $6.42 \pm 3.34$ & $5.83 \pm 3.27$ & $6.91 \pm 2.88$ & $7.91 \pm 1.38$ & 0.665 \\
\hline General health & $6.33 \pm 1.44$ & $6.50 \pm 1.57$ & $6.45 \pm 2.62$ & $7.91 \pm 1.81^{* *}$ & $0.041^{\dagger}$ \\
\hline Global score of health perception & $3.71 \pm 0.95$ & $4.10 \pm 1.16$ & $4.36 \pm 1.47$ & $5.39 \pm 1.16^{* * *}$ & 0.099 \\
\hline
\end{tabular}

Values are presented as means $\pm \mathrm{SD}$. The total number of subjects is $23 .^{*} p<0.05 .{ }^{* *} p<0.01 .{ }^{* * *} p<0.001$. Time $\times$ group interaction ${ }^{\dagger} p<0.05$.

The SF-36 physical ( $p<0.001$ in the ECC group and $p<0.01$ in the CON group) and GH scores $(p<0.01)$ increased significantly in both groups, while PF and the RP items were increased only in the ECC group ( $p<0.05$ and $p<0.01$, respectively). Data related to the changes in SF-36 scores are summarized in Table 3. Correlation tests did not reveal any strong relation between variations of the anthropometric characteristics, body composition, functional parameters, and SF-36 scores (Fig. 1).

The perceived physical condition item increased significantly in both groups $(p<0.001$ in the ECC group and $p<0.05$ in the CON group). The global HP score $(p<0.001)$ and the perceived general health item $(p<0.01)$ increased only in the ECC group. Data related to the changes in HP scores are summarized in Table 4. Correlation tests did not reveal any strong correlation between variations of the anthropometric characteristics, body composition, functional parameters, and HP scores (Fig. 1). 
Julian et al.: ECC Training and Quality of Life

\section{Discussion/Conclusion}

Although both ECC and CON training improved the quality of life, anthropometric, body composition, and functional parameters, ECC training, which induces a greater adiposity reduction and muscle mass increase [results previously discussed in 9], seems to be also more effective in increasing HRQOL and HP compared to CON training. Nevertheless, these improvements are not likely to be explained due to weight loss, body composition changes, aerobic capacities, or strength variations.

The observed improved HRQOL after both exercise trainings is fully in line with the literature which has demonstrated that various interventional programs incorporating physical activity with or without dietary counselling increase HRQOL in children with obesity [21,22]. This is of particular interest because children and adolescents with obesity have a significantly lower HRQOL compared to lean individuals, not only in terms of the overall quality of life but also in terms of its physical, psychological, social, and school domains [23]. While the results of the VSP-A questionnaire showed improvements in both physical and psychological dimensions, only the physical domain was found to be positively changed according to the SF-36 questionnaire. This is concordant with previous studies showing improvements in both physical and psychological dimensions of HRQOL in response to weight loss interventions [24-26], while other studies have suggested improved physical rather than psychological dimensions $[27,28]$. Moreover, we observed a major improvement in the body image item $(\sim 80 \%)$, which is of great interest because body image is associated with self-esteem and mood in adolescents and implicated in the induction of eating disorders $[29,30]$.

While most of the existing literature questions the effects of multidisciplinary interventions on quality of life, our study is the first to evaluate the impact of ECC-based physical training on HRQOL. HRQOL improvement after ECC training is of principal interest because it has been proposed as a promising approach for the prevention and treatment of most chronic diseases [12], particularly for overweight and obese individuals [9, 10]. Moreover, even though every exercise modality can lead to exercise-induced muscle damage, ECC is more likely to induce muscle damage compared to CON training. Indeed, ECC exercise has more damaging effects due to the higher force developed, with overstretching causing mechanical disruption of muscle fibers and temporary lesions of the cytoskeleton [31]. The inflammatory response that induces ulterior protective adaptation of the muscles is thus responsible for delayed onset muscle soreness that could temporarily alter exercise tolerance, muscle strength, and range of movements [31] and thus potentially influencing the physical aspects of quality of life. This is of particular importance since patients with obesity display more significant and more prolonged exercise-induced muscle damage after ECC exercise than lean patients [32]. Importantly, the increased HRQOL observed in response to ECC training, and particularly the major improvement in physical well-being and energy-vitality items, supports the good tolerance of that type of training modality in adolescents with obesity (and that might favor training adherence and a higher motivation to continue physical activity after the intervention). This tolerance can be explained by the progressive increase in the load and time of exercise and the continuous professional and individualized supervision, which favored a reduction of both the intensity and the duration of delayed-onset muscle soreness $[10,13]$. Moreover, ECC intervention also induced a significant improvement in HP (while it remained unchanged in the CON group), particularly in physical and general HP items. Although HP has been previously associated with health indicators and physical fitness in both normal-weight adults and adults with obesity $[18,19,33]$, it remains less studied in adolescents with obesity. Khammassi et al. [20] are, to our knowledge, the first to interrogate HP in adolescents with obesity, showing improvement of its global score as well as physical, adiposity, alimentation, and general health items in response to a 10-month

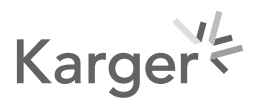


multidisciplinary weight-loss program, which is consistent with the present results observed in the ECC group only. The greater HRQOL and HP score improvements in the ECC group compared to the CON group are in addition to greater adiposity reduction, strength gain, and metabolic improvements, as previously observed [10].

Except for the increase in energy-vitality that was associated with decreased weight, BMI, and fat mass, none of the HRQOL and HP improvements were significantly associated with anthropometric, body composition, and physical capacities changes. Thus, the greater HRQOL improvement observed in the ECC group compared to the CON group does not seem to be related to the previously documented higher effectiveness of ECC training on body composition and functional capacities [10]. In the literature, the potential associations between induced body weight and body composition improvements with the observed HRQOL changes are debatable. While most studies describe negative associations between HRQOL and obesity indicators (body weight, BMI, and fat mass percentage) $[2,5,8]$, several recent interventional studies have failed to observe any relationship between these indicators and HRQOL in young patients. Rank et al. [7] showed that a 4- to 6-week multidisciplinary weight loss intervention induced improved HRQOL independently of body composition changes. Similarly, Hoedjes et al. [6] showed that a 1-year inpatient lifestyle treatment improved the overall HRQOL and that this improvement was maintained 1 year after treatment despite a partial weight regain, suggesting that antiobesity interventions improved HRQOL independently of body weight and body composition changes. In a similar population, recent results from Khammassi et al. [20] also failed to observe any association between improved HRQOL and HP and body weight, fat mass, and fat-free mass changes after a 10-month multidisciplinary intervention. Some studies, however, have suggested a positive correlation between HRQOL and anthropometric but not with cardiorespiratory fitness responses to weight loss [34]. Interestingly, Evaristo et al. [35] showed that physical fitness but not body composition or body mass modifications mediated HRQOL changes in response to weight loss in children with obesity. The present results do not seem to support this association since we failed to observe any association between the improvements and changes in HRQOL and HP with both our aerobic and our anaerobic fitness indicators. Previous studies have suggested that the lack of an association between HRQOL and body composition changes could explain the commonly observed maintained HRQOL improvement despite weight regain several months after weight loss interventions [6, 24, 36]. The central effects of cardiorespiratory fitness could partly explain this lack of an association. Indeed, recent neuroimaging studies in children with obesity have shown that cardiorespiratory fitness (independent of BMI) induce greater grey and white matter volume of numerous brain structures, which are engaged in attention, memory, and cognitive performance, and particularly the hippocampus, which is implicated in memory, anxiety, and mood [37].

The present results must be considered and interpreted in light of some limitations. First, QOL includes dynamic components such as changes in family situation, school, or social context, which are partly biased due to the supervised nature of our medical center and the short duration of the intervention (12 weeks). Nevertheless, self-reported questionnaires of HRQOL are subjective evaluations, which can be subject to perception bias. Concerning the relatively small sample size, the number of patients recruited was preliminarily fixed by a sample size estimation based on previous literature relative to the effect of ECC training on body composition parameters and involving 10-15 individuals per group [38]. Moreover, it would have been interesting to conduct a follow-up assessment to analyze whether the observed changes are maintained over time after the training programs, which has not been possible here for practical reasons.

The present work is the first to evaluate the impact of ECC training on HRQOL and provides new insights regarding the effect of the training modality on both HRQOL and HP in

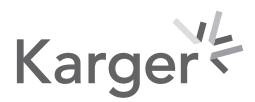




\section{Obesity Facts}

adolescents with obesity. We showed that the 2 training modalities performed at the same $\mathrm{VO}_{2}$ improved HRQOL. Though associated, these improvements may not be explained by changes in weight loss, body composition, aerobic capacity, or strength. ECC training induced a greater gain in HRQOL compared to CON training, which does not seem related to the previously documented higher effectiveness of ECC training on body composition. While these results seem to reinforce that ECC exercise is an effective strategy to treat pediatric obesity, further work is required to confirm and encourage its clinical use.

\section{Acknowledgement}

The authors thank all of the individuals who participated in this study, as well as the Pediatric Obesity Center (Tza Nou, la Bourboule, France), the Observatoire National de l'Activité Physique et de la Sédentarité (ONAPS), and the Comité Régional des Offices Municipaux du Sport d'Auvergne Rhône-Alpes-Auvergne (France).

\section{Statement of Ethics}

All of the adolescents and their parents provided written informed consent after being fully briefed and advised about the study procedures. This trial was approved by the relevant Ethical Committee (Comité de Protection des Personnes Est IV) and is registered with ClinicalTrials.gov (NCT02925572). It was conducted in compliance with the recommendations for good clinical practice and the Declaration of Helsinki.

\section{Conflict of Interest Statement}

The authors have no conflict of interests to declare.

\section{Funding Sources}

This work was supported by grants from the Centre National pour le Développement du Sport and from the University Teaching Hospital of Clermont-Ferrand (AOI2015).

\section{Author Contributions}

V.J., D.T., R.R., and M.D. conceived and designed the research. V.J., D.T., and M.M. conducted the experiments. B.P. and C.L. contributed the statistical analyses. V.J., D.T., and B.P. analyzed the data. V.J. and D.T. wrote this paper. All of the authors read and approved this work.

\section{Karger'א}


Julian et al.: ECC Training and Quality of Life

\section{References}

1 Daniels SR, Arnett DK, Eckel RH, Gidding SS, Hayman LL, Kumanyika S, et al. Overweight in children and adolescents: pathophysiology, consequences, prevention, and treatment. Circulation. 2005 Apr; 111(15): 1999-2012.

2 Tsiros MD, Olds T, Buckley JD, Grimshaw P, Brennan L, Walkley J, et al. Health-related quality of life in obese children and adolescents. Int J Obes (Lond). 2009 Apr;33(4):387-400.

3 Jeffery RW, Wing RR, Sherwood NE, Tate DF. Physical activity and weight loss: does prescribing higher physical activity goals improve outcome? Am J Clin Nutr. 2003 Oct; 78(4):684-9.

4 Dankel SJ, Loenneke JP, Loprinzi PD. Health Outcomes in Relation to Physical Activity Status, Overweight/ Obesity, and History of Overweight/Obesity: A Review of the WATCH Paradigm. Sports Med. 2017 Jun;47(6): 1029-34.

5 Fonvig CE, Hamann SA, Nielsen TR, Johansen Mø, Grønbæk HN, Mollerup PM, et al. Subjective evaluation of psychosocial well-being in children and youths with overweight or obesity: the impact of multidisciplinary obesity treatment. Qual Life Res. 2017 Dec;26(12):3279-88.

6 Hoedjes M, Makkes S, Halberstadt J, Noordam H, Renders CM, Bosmans JE, et al. Health-Related Quality of Life in Children and Adolescents with Severe Obesity after Intensive Lifestyle Treatment and at 1-Year Follow-Up. Obes Facts. 2018;11(2):116-28.

7 Rank M, Wilks DC, Foley L, Jiang Y, Langhof H, Siegrist M, et al. Health-related quality of life and physical activity in children and adolescents 2 years after an inpatient weight-loss program. J Pediatr. 2014 Oct;165(4): 732-7.e2.

8 Morrison KM, Shin S, Tarnopolsky M, Taylor VH. Association of depression \& health related quality of life with body composition in children and youth with obesity. J Affect Disord. 2015 Feb;172:18-23.

9 Julian V, Thivel D, Costes F, Touron J, Boirie Y, Pereira B, et al. Eccentric Training Improves Body Composition by Inducing Mechanical and Metabolic Adaptations: A Promising Approach for Overweight and Obese Individuals. Front Physiol. 2018 Aug; 9:1013.

10 Julian V, Thivel D, Miguet M, Pereira B, Costes F, Coudeyre E, et al. Eccentric cycling is more efficient in reducing fat mass than concentric cycling in adolescents with obesity. Scand J Med Sci Sports. 2019 Jan;29(1):4-15.

11 Paschalis V, Nikolaidis MG, Giakas G, Theodorou AA, Sakellariou GK, Fatouros IG, et al. Beneficial changes in energy expenditure and lipid profile after eccentric exercise in overweight and lean women. Scand J Med Sci Sports. 2010 Feb;20(1):e103-11.

12 Hoppeler H. Moderate Load Eccentric Exercise; A Distinct Novel Training Modality. Front Physiol. 2016 Nov; 7:483.

13 LaStayo P, Marcus R, Dibble L, Frajacomo F, Lindstedt S. Eccentric exercise in rehabilitation: safety, feasibility, and application. J Appl Physiol (1985). 2014 Jun;116(11):1426-34.

14 Cole TJ, Bellizzi MC, Flegal KM, Dietz WH. Establishing a standard definition for child overweight and obesity worldwide: international survey. BMJ. 2000 May;320(7244):1240-3.

15 Sapin C, Simeoni MC, El Khammar M, Antoniotti S, Auquier P. Reliability and validity of the VSP-A, a healthrelated quality of life instrument for ill and healthy adolescents. J Adolesc Health. 2005 Apr;36(4):327-36.

16 Simeoni MC, Auquier P, Antoniotti S, Sapin C, San Marco JL. Validation of a French health-related quality of life instrument for adolescents: the VSP-A. Qual Life Res. 2000;9(4):393-403.

17 Garratt AM, Ruta DA, Abdalla MI, Buckingham JK, Russell IT. The SF36 health survey questionnaire: an outcome measure suitable for routine use within the NHS? BMJ. 1993 May;306(6890):1440-4.

18 Garnier S, Gaubert I, Joffroy S, Auneau G, Mauriège P. Impact of brisk walking on perceived health evaluated by a novel short questionnaire in sedentary and moderately obese postmenopausal women. Menopause. 2013 Aug;20(8):804-12.

19 Genin PM, Degoutte F, Finaud J, Pereira B, Thivel D, Duclos M. Effect of a 5-Month Worksite Physical Activity Program on Tertiary Employees Overall Health and Fitness. J Occup Environ Med. 2017 Feb;59(2):e3-10.

20 Khammassi M, Miguet M, O'Malley G, Fillon A, Masurier J, Damaso AR, et al. Health-related quality of life and perceived health status of adolescents with obesity are improved by a 10-month multidisciplinary intervention. Physiol Behav. 2019 Oct;210:112549.

21 Freitas CR, Gunnarsdottir T, Fidelix YL, Tenório TR, Lofrano-Prado MC, Hill JO, et al. Effects of a psychological intervention on the quality of life of obese adolescents under a multidisciplinary treatment. J Pediatr (Rio J). 2017 Mar - Apr;93(2):185-91.

22 Morano M, Rutigliano I, Rago A, Pettoello-Mantovani M, Campanozzi A. A multicomponent, school-initiated obesity intervention to promote healthy lifestyles in children. Nutrition. 2016 0ct;32(10):1075-80.

23 Eliacik K, Bolat N, Koçyiğit C, Kanik A, Selkie E, Yilmaz H, et al. Internet addiction, sleep and health-related life quality among obese individuals: a comparison study of the growing problems in adolescent health. Eat Weight Disord. 2016 Dec;21(4):709-17.

24 Blissmer B, Riebe D, Dye G, Ruggiero L, Greene G, Caldwell M. Health-related quality of life following a clinical weight loss intervention among overweight and obese adults: intervention and 24 month follow-up effects. Health Qual Life Outcomes. 2006 Jul;4(1):43.

25 Knöpfli BH, Radtke T, Lehmann M, Schätzle B, Eisenblätter J, Gachnang A, et al. Effects of a multidisciplinary inpatient intervention on body composition, aerobic fitness, and quality of life in severely obese girls and boys. J Adolesc Health. 2008 Feb;42(2):119-27. 
26 Ravens-Sieberer U, Bullinger M. Assessing health-related quality of life in chronically ill children with the German KINDL: first psychometric and content analytical results. Qual Life Res. 1998 Jul;7(5):399-407.

27 Fine JT, Colditz GA, Coakley EH, Moseley G, Manson JE, Willett WC, et al. A prospective study of weight change and health-related quality of life in women. JAMA. 1999 Dec;282(22):2136-42.

28 Warkentin LM, Das D, Majumdar SR, Johnson JA, Padwal RS. The effect of weight loss on health-related quality of life: systematic review and meta-analysis of randomized trials. Obes Rev. 2014 Mar;15(3):169-82.

29 Gall K, van Zutven K, Lindstrom J, Bentley C, Gratwick-Sarll K, Harrison C, et al. Obesity and emotional wellbeing in adolescents: roles of body dissatisfaction, loss of control eating, and self-rated health. Obesity (Silver Spring). 2016 Apr;24(4):837-42.

30 Paxton SJ, Neumark-Sztainer D, Hannan PJ, Eisenberg ME. Body dissatisfaction prospectively predicts depressive mood and low self-esteem in adolescent girls and boys. J Clin Child Adolesc Psychol. 2006 Dec; 35(4):539-49.

31 Douglas J, Pearson S, Ross A, McGuigan M. Eccentric Exercise: Physiological Characteristics and Acute Responses. Sports Med. 2017 Apr;47(4):663-75.

32 Paschalis V, Nikolaidis MG, Theodorou AA, Deli CK, Raso V, Jamurtas AZ, et al. The effects of eccentric exercise on muscle function and proprioception of individuals being overweight and underweight. J Strength Cond Res. 2013 Sep;27(9):2542-51.

33 Genin PM, Dessenne P, Finaud J, Pereira B, Thivel D, Duclos M. Health and Fitness Benefits But Low Adherence Rate: Effect of a 10-Month Onsite Physical Activity Program Among Tertiary Employees. J Occup Environ Med. 2018 Sep;60(9):e455-62.

34 Carlone Baldino Garcia N, Lopes WA, Locateli JC, Ferraz Simões C, de Oliveira GH, de Souza Mendes VH, et al. Multidisciplinary obesity treatment program improved health-related quality of life and positively correlated with anthropometric and body composition but not with cardiorespiratory fitness parameters in adolescents. Qual Life Res. 2019 Jul;28(7):1803-12.

35 Evaristo S, Moreira C, Santos R, Lopes L, Abreu S, Agostinis-Sobrinho C, et al. Associations between healthrelated quality of life and body mass index in Portuguese adolescents: LabMed physical activity study. Int J Adolesc Med Health. 2018 Feb;31(5):/j/ijamh.2019.31.issue-5/ijamh-2017-0066/ijamh-2017-0066.xml.

36 Fontaine KR, Barofsky I, Andersen RE, Bartlett SJ, Wiersema L, Cheskin LJ, et al. Impact of weight loss on health-related quality of life. Qual Life Res. 1999 May;8(3):275-7.

37 Esteban-Cornejo I, Rodriguez-Ayllon M, Verdejo-Roman J, Cadenas-Sanchez C, Mora-Gonzalez J, ChaddockHeyman L, et al. Physical Fitness, White Matter Volume and Academic Performance in Children: Findings From the ActiveBrains and FITKids2 Projects. Front Psychol. 2019 Feb;10:208.

38 Mueller M, Breil FA, Vogt M, Steiner R, Lippuner K, Popp A, et al. Different response to eccentric and concentric training in older men and women. Eur J Appl Physiol. 2009 Sep;107(2):145-53. 